\title{
Serial QuantiFERON-TB Gold In-Tube assay and tuberculin skin test to diagnose latent tuberculosis in household Mexican contacts: conversion and reversion rates and associated factors using conventional and borderline zone definitions
}

\author{
Joel Monárrez-Espino', José Antonio Enciso-Moreno², \\ Lucie Laflamme', Carmen J Serrano ${ }^{2 /+}$ \\ ${ }^{1}$ Department of Public Health Sciences, Karolinska Institute, Stockholm, Sweden \\ ${ }^{2}$ Medical Research Unit Zacatecas, Mexican Institute of Social Security, Zacatecas, Mexico
}

\begin{abstract}
A cohort of 123 adult contacts was followed for 18-24 months (86 completed the follow-up) to compare conversion and reversion rates based on two serial measures of QuantiFERON (QFT) and tuberculin skin test (TST) (PPD from TUBERSOL, Aventis Pasteur, Canada) for diagnosing latent tuberculosis (TB) in household contacts of TB patients using conventional (C) and borderline zone (BZ) definitions. Questionnaires were used to obtain information regarding TB exposure, TB risk factors and socio-demographic data. QFT (IU/mL) conversion was defined as $<0.35$ to $\geq 0.35$ (C) or $<0.35$ to $>0.70$ (BZ) and reversion was defined as $\geq 0.35$ to $<0.35$ (C) or $\geq 0.35$ to $<0.20$ (BZ); TST (mm) conversion was defined as $<5$ to $\geq 5$ (C) or $<5$ to $>10$ (BZ) and reversion was defined as $\geq 5$ to $<5$ (C). The QFT conversion and reversion rates were $10.5 \%$ and $7 \%$ with $C$ and $8.1 \%$ and $4.7 \%$ with the $B Z$ definitions, respectively. The TST rates were higher compared with QFT, especially with the C definitions (conversion 23.3\%, reversion 9.3\%). The QFT conversion and reversion rates were higher for TST $\geq 5$; for TST, both rates were lower for $Q F T<0.35$. No risk factors were associated with the probability of converting or reverting. The inconsistency and apparent randomness of serial testing is confusing and adds to the limitations of these tests and definitions to follow-up close TB contacts.
\end{abstract}

Key words: close contact - QuantiFERON - latent TB - Mexico - serial testing

The household contacts of patients diagnosed with active tuberculosis (TB) are at higher risk of contagion and of developing active disease in a shorter period of time compared with sporadic contacts (Fox et al. 2013). The relatively large incidence rate of TB infection $(\sim 1,500$ per 100,000 person-years) reported by contacts of TB patients from low- and middle-income countries during the first year after exposure to the index case emphasises the importance of serial screening, particularly among individuals not receiving prophylactic treatment. It has been reported that $51.5 \%$ of all contacts $[95 \%$ confidence interval (CI) 47.1-55.8\%] have a tuberculin skin test (TST) that is positive for latent TB infection (LTBI) (Fox et al. 2013).

Serial TST testing for LTBI has been proposed for people constantly exposed to infected individuals, such as household members or health workers in contact with TB patients. However, repeated TSTs to diagnose LTBI in high-risk groups have been difficult to interpret because

doi: 10.1590/0074-0276140085

Financial support: CONACYT-SALUD-2008-C01-87657,

Program for international cooperation CIS of the IMSS

+ Corresponding author: carmenyuyu2000@yahoo.com.mx

Received 6 March 2014

Accepted 19 August 2014 the findings show inexplicable reversions (i.e., decrease in skin induration size from a prior positive test) or unclear conversions (i.e., increase in induration size from an earlier negative test) resulting from either random variability (e.g., differences in administration, reading or biologic response), boosting (i.e., immunologic recall of pre-existing delayed hypersensitivity to mycobacterial antigens) or an actual new infection (Menzies 1999).

Interferon-gamma release assays (IGRAs) are alternatives to TSTs (Mazurek et al. 2005), although findings suggest that reversions and conversions are also frequent (Ewer et al. 2006, Friedman et al. 2006, Pai et al. 2006, Franken et al. 2007, Hill et al. 2007a, b, Lee et al. 2008, Slater et al. 2013, Dorman et al. 2014) and there is no consensus on how to define and interpret these phenomena (Pai \& O’Brien 2007, Pai et al. 2007, Slater et al. 2013, Dorman et al. 2014).

Large clinical cohorts are currently being conducted in the United Kingdom (PREDICT study) and Europe (TBNET study) to attempt to overcome some of the limitations of the IGRAs as tests for diagnosing LTBI (Abubakar et al. 2013). The most updated guidelines address such limitations by using more flexible criteria [e.g., modified cut-offs according to the person or population tested and borderline zones (BZs) to define conversion and reversion] aimed at improving the sensitivity and specificity of the assays (Mazurek et al. 2010, Abubakar et al. 2012, Moon \& Hur 2013). 
Serial testing using IGRAs and/or TST to diagnose LTBI among household adult contacts of TB patients has not been reported in Mexico or Latin America; therefore, conversion and reversion rates in these populations remain unknown and very limited evidence exists on factors associated with their stability over time. Moreover, few studies worldwide have compared the reversion and conversion rates for LTBI based on TSTs and IGRAs within the critical first years after exposure using household contacts (Pai et al. 2009, Shah et al. 2011) and no study has tried to explore whether these rates relate to personal or exposure characteristics.

We previously reported a high baseline prevalence of LTBI using the TST ( $\geq 5 \mathrm{~mm} 53.6 \%$ ) and QuantiFERON-TB Gold In-Tube assay (QFT-GIT) $(\geq 0.35 \mathrm{IU} /$ $\mathrm{mL}$ 41.4\%) (Cellestis Inc, USA) from a cohort of 123 adult contacts in central Mexico (Serrano-Escobedo et al. 2013). Herein, we determine the performance characteristics of the QFT-GIT vs. TST for serial testing of those household contacts. After an 18-month follow-up period, we demonstrate and compare the QFT-GIT and TST responses in terms of conversions and reversions using conventional and BZ cut-off definitions from two serial measures. In addition, this study explores the epidemiological associations between constitutional, sociodemographic and exposure factors and the occurrence rates of conversions and reversions.

\section{PATIENTS, MATERIALS AND METHODS}

Study design - This study used a cohort of 123 adult contacts of 39 TB patients who were followed up for 18 months. The index cases were diagnosed with pulmonary TB (positive acid-fast bacilli smear and/or culture) between 2008-2010 in the central Mexican state of Zacatecas, according to the Ministry of Health. Baseline data were used to report on the performance of the QFTGIT and TST to identify LTBI among these contacts (Serrano-Escobedo et al. 2013).

Trained staff interviewed the TB patients and contacts at their homes using structured questionnaires. Basic socio-demographic data and known risk factors for TB were obtained. Furthermore, information concerning the type and number of contacts, along with the typical frequency and duration of the interaction between index cases and contacts, was recorded from TB patients during the first visit and from contacts a few months later. The QFT-GIT and TST measurements were taken at baseline from all the contacts. Thereafter, the contacts were located after 18-24 months (between March-May 2012) to obtain follow-up QFT-GIT and TST data.

QFT-GIT and TST measurements - QFT-GIT and TST analyses were requested from contacts and were performed using identical procedures and protocols at baseline and follow-up. However, endpoint data were unavailable for analyses in 37 contacts. The main reasons for loss to follow-up were refusals to participate $(\mathrm{n}=21)$ and our inability to locate individuals $(\mathrm{n}=10)$ during the followup testing. Other reasons for excluding contacts from the analyses included one person who developed pulmonary TB, two women who became pregnant, one person who was not retested due to an initial side effect, one person who was hospitalised for reasons unrelated to TB and one person who died during the follow-up period.

Full details regarding the testing and measuring procedures used for the QFT-GIT and TST were described earlier (Serrano-Escobedo et al. 2013). Briefly, QFT-GIT testing occurred prior to the TST application; values of $\geq 0.35 \mathrm{IU} / \mathrm{mL}$ after adjustment with a negative control were considered positive. The TST was based on the Mantoux method; a transverse skin induration diameter $\geq 5 \mathrm{~mm}$ was considered positive.

Definitions of conversion and reversion - Various cut-offs were used to define conversion (i.e., negative to positive result) and reversion (i.e., positive to negative result) according to both tests and to the QFT-GIT and TST measures independently.

For the QFT-GIT (in IU/mL), conversion was defined as follows: (i) conventional cut-off, from $<0.35$ to $\geq 0.35$ and (ii) BZ cut-off, from $<0.35$ to $>0.70$. Reversion was defined as: (i) conventional cut-off, from $\geq 0.35$ to $<0.35$ and (ii) from $\geq 0.35$ to $<0.20$. These thresholds were determined according to recent recommendations (Ringshausen et al. 2012, Whitworth et al. 2012).

For the TST (in mm induration), conversion was defined as follows: (i) conventional cut-off, from $<5$ at baseline to $\geq 5$ at follow-up and (ii) BZ cut-off, from $<$ 5 to $>10$; this $6 \mathrm{~mm}$ increment, which was suggested to overcome random variations, was used to create a corresponding TST borderline definition to be compared with that of the QFT-GIT; reversion was defined conventionally as $\geq 5$ at baseline to $<5 \mathrm{~mm}$ at follow-up.

Basic socio-demographic and exposure data - A relatively large amount of data was obtained from each of the contacts included in this cohort. However, only selected personal [sex, age group and body mass index (BMI)], exposure (exposure time and type of contact) and risk [Bacillus Calmette-Guerin (BCG) scar] factors for TB were used in the stratified results presented herein.

The exposure time was computed using the number of hours that each contact was exposed to the TB patient according to the questionnaire applied to the index case. The total time was calculated from a set of questions referring to the symptomatic period when the person had not been treated (i.e., the contagious period). This variable was dichotomised as $1-500 \mathrm{~h}$ and $>500 \mathrm{~h}$ within the first three-month symptomatic period.

The type of contact with the index case was calculated from the variables contained in the questionnaire applied to the contacts after the index case was treated (i.e., the non-contagious period). The information was categorised as very close (5-7 days per week with $\geq 30 \mathrm{~h}$ per week), close (5-7 days per week with 21-30 h per week), irregular (1-4 days per week with 6-20 h per week) and occasional (1-4 days per week with $<6 \mathrm{~h}$ per week).

Statistical analysis - Frequencies and proportions were used to present the categorical data and chi-square tests were used to establish differences between the contacts who were and were not followed up. Mann-Whitney $U$ tests were used to assess median and distribution differences between groups for continuous data not normally distributed. 
Kappa coefficients were used to measure agreements between the QFT-GIT and TST using the conventional dichotomous definition to diagnose LTBI at baseline.

The conversion and reversion rates occurring during the follow-up period were compared for both measures using the conventional and BZ cut-offs that were defined. The proportions of conversion and reversion of the QFT-GIT and TST using the BZ cut-offs were stratified according to sex, age group, BMI group, BCG scar and exposure categories.

Finally, exploratory multinomial regression analyses were used to identify risk factors for conversion and reversion using the category "remained positive/negative" at the endpoint as the reference. Four adjusted models were run using the QFT-GIT and TST with conventional and $\mathrm{BZ}$ definitions as dependent variables. The independent variables included the previously referenced variables and the initial QFT-GIT and TST status. Other baseline characteristics of the contacts and index cases not detailed here (e.g., morbidity, income, household conditions, civil status, literacy, schooling, smoking, alcohol use, migration etc.) were also tested. Crude and adjusted odds ratios with $95 \%$ CIs were computed.

The data were entered and analysed using IBM $^{\circledR}$ SPSS $^{\circledR}$ v.22 (IBM, USA). Statistical significance was set at $\mathrm{p}<0.05$.

Ethics - The research protocol was developed in accordance with the Helsinki Declaration and was revised and approved by the National Health Research and Ethical Committee at the Mexican Institute of Social Security (ID R-2008-3301-15). All the participants provided written informed consent.

\section{RESULTS}

Figure illustrates the study design for the cohort of contacts that was followed. From the initial 123 contacts recruited, QFT-GIT and TST data were available for 86 individuals (follow-up rate of $70 \%$ ). The agreement and kappa coefficients between the QFT-GIT and TST were $72.3 \%$ and $0.45 \%$ at baseline and $68.6 \%$ and $0.41 \%$ at the endpoint, respectively. Although the majority of the contacts lost to follow-up had positive $(\mathrm{n}=17)$ or negative $(\mathrm{n}$ $=12$ ) results in both measures, the subjects with positive QFT-GIT and negative TST at baseline had the lowest response rate $(55.6 \%)$.

Table I compares selected characteristics between contacts who completed follow-up and contacts who were not followed. Although no statistically significant differences were identified, the persons lost to follow-up had slightly higher median BMI values (26 vs. $25 \mathrm{~kg} /$ $\left.\mathrm{m}^{2} ; \mathrm{p}=0.09\right)$, higher QFT-GIT levels ( 0.15 vs. $0.04 \mathrm{IU} /$ $\mathrm{mL} ; \mathrm{p}=0.12)$ and lower median TST indurations ( 3 vs. 7 $\mathrm{mm} ; \mathrm{p}=0.13$ ) compared with those who were followed. No clear differences in the exposure time to the TB patient were detected, either as referred by the index case or the contacts, although a higher proportion of contacts exposed for $>500 \mathrm{~h}$ was observed in the subjects lost to follow-up (37.8 vs. $22.1 \%$ ).

The conversion and reversion rates using the conventional and BZ definitions are listed in Table II. Consistent with expectations, the rates were lower when the

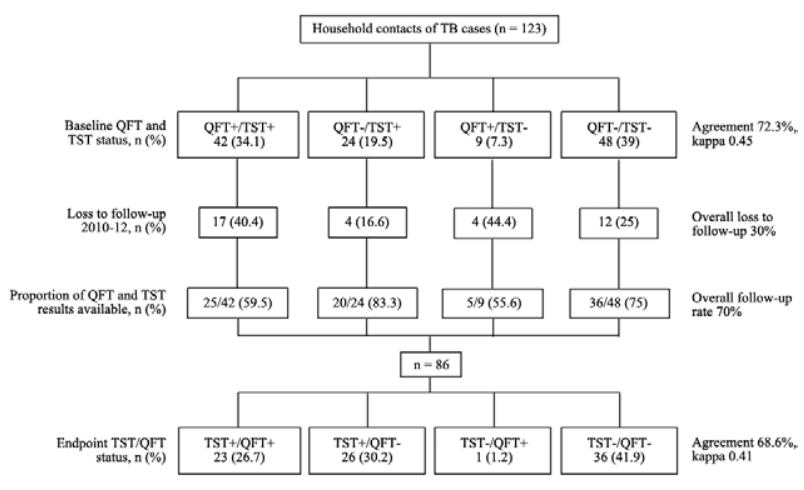

Flow-chart of the Zacatecas, Mexico cohort for household contacts of tuberculosis (TB) patients followed-up between 2010-2012 using QuantiFERON (QFT) (positive if $\geq 0.35 \mathrm{UI} / \mathrm{mL}$ ) and tuberculin skin test (TST) (positive if $\geq 5 \mathrm{~mm}$ induration) at baseline and endpoint.

borderline thresholds were used. For the QFT-GIT, the respective overall proportions of conversions and reversions were $10.5 \%$ and $7 \%$ when the conventional definition was used and $8.1 \%$ and $4.7 \%$ according to the $\mathrm{BZ}$ criterion. The conversion and reversion rates using TST were notably higher than with the QFT-GIT, especially when the conventional cut-offs were used $(23.3 \%$ and $9.3 \%$, respectively). The rates varied depending on the corresponding baseline values of the TST or QFT-GIT. For the QFT-GIT, the conversion and reversion rates were higher if the baseline TST was $\geq 5 \mathrm{~mm}$, whereas for the TST, the rates tended to be lower when the QFTGIT was $<0.35 \mathrm{IU} / \mathrm{mL}$ at baseline.

Differences in the proportions of conversions and reversions using the BZ cut-offs for the QFT-GIT and TST stratified by selected factors are shown in Table III. Although some proportions differed between groups, no significant differences were detected for any of the variables assessed. For the QFT-GIT, the majority of the seven conversions occurred in men $(71.4 \%)$ and in people vaccinated against TB (71.4\%). The results by the type and total hours of exposure between TB patients and contacts were inconsistent; although the majority of conversions occurred in contacts with shorter exposure times as referred by the index case, the contacts with close or very close interactions had higher conversion rates according to the contacts. Four reversions were observed and the patterns also differed between categories, with the majority of reversions occurring in contacts with normal BMIs (75\%), with BCG scars $(75 \%)$ and with close or very close interactions with the TB patients $(75 \%)$. Using the TST criteria, the majority of the conversions $(71.4 \%)$ occurred in women, in persons with normal BMIs (71.4\%); in all seven conversions, the contacts had BCG scars. Although less clear, the findings were also inconsistent with regards to exposure to the index case. The eight reversions occurred in women in very close or close contact with the TB patient. The majority of these subjects were overweight $(62.5 \%)$ and had received $B C G$ vaccinations (75\%).

None of the factors explored in the crude or adjusted multinomial regression models was statistically significant, irrespective of the dependent variable assessed 
TABLE I

Selected characteristics of the household contacts of tuberculosis patients at baseline stratified by follow-up status, Zacatecas cohort, Mexico, 2010-2012

\begin{tabular}{|c|c|c|c|c|}
\hline Characteristic & Category & $\begin{array}{l}\text { Followed-up } \\
\qquad \begin{array}{c}(\mathrm{n}=86) \\
\mathrm{n}(\%)\end{array}\end{array}$ & $\mathrm{p}^{a}$ & $\begin{array}{l}\text { Loss to follow-up } \\
\qquad \begin{array}{c}(\mathrm{n}=37) \\
\mathrm{n}(\%)\end{array}\end{array}$ \\
\hline \multirow[t]{2}{*}{ Sex } & Male & $34(39.5)$ & 0.50 & $17(45.9)$ \\
\hline & Female & $52(60.5)$ & & $20(54.1)$ \\
\hline Body mass index & Median (range) & $25.1(18.2-37.3)$ & 0.09 & $26.0(20.3-38.2)$ \\
\hline \multirow[t]{5}{*}{ Age (years) } & $<30$ & $24(27.9)$ & 0.89 & $11(29.7)$ \\
\hline & $30-39$ & $19(22.1)$ & & $10(27)$ \\
\hline & $40-49$ & $15(17.4)$ & & $5(13.5)$ \\
\hline & $\geq 50$ & $28(32.6)$ & & $11(29.7)$ \\
\hline & Median (range) & $39.5(17.1-87.4)$ & 0.51 & $36.4(18.2-74.4)$ \\
\hline \multirow[t]{2}{*}{ BCG scar } & Yes & $75(87.2)$ & 0.61 & $31(83.8)$ \\
\hline & No & $11(12.8)$ & & $6(16.2)$ \\
\hline \multirow[t]{3}{*}{ Total exposure time ${ }^{b}$ (hours) } & $1-500$ & $40(46.5)$ & 0.19 & $14(37.8)$ \\
\hline & $>500$ & $19(22.1)$ & & $14(37.8)$ \\
\hline & Undetermined & $27(31.4)$ & & $9(24.3)$ \\
\hline \multirow[t]{2}{*}{ Type of contact $^{c}$} & Close or very close & $68(79.1)$ & 0.79 & $30(81.1)$ \\
\hline & Irregular or occasional & $18(20.9)$ & & 7 (18.9) \\
\hline \multirow[t]{4}{*}{ TST (mm) } & $0-4$ & $36(41.9)$ & 0.30 & $21(56.8)$ \\
\hline & $5-9$ & $17(19.8)$ & & $6(16.2)$ \\
\hline & $>10$ & $33(38.4)$ & & $10(27)$ \\
\hline & Median (range) & $7(0-28)$ & 0.13 & $3(0-20)$ \\
\hline \multirow{3}{*}{ QFT (IU/mL) } & $<0.35$ & $52(60.5)$ & 0.70 & $21(56.8)$ \\
\hline & $\geq 0.35$ & $34(39.5)$ & & $16(43.2)$ \\
\hline & Median (range) & $0.04(0-10)$ & 0.12 & $0.15(0-10)$ \\
\hline
\end{tabular}

$a$ : Pearson chi-square (nominal data) and Mann-Whitney $U$ tests (not normally distributed continuous data) were used; $b$ : computed from a questionnaire applied to the index case when symptomatic and untreated (contagious period); $c$ : calculated from a questionnaire applied to the contacts after the index case was treated (non-contagious period: very close, 5-7 days/week with $\geq$ $30 \mathrm{~h} /$ week, close, 5-7 days/week with 21-30 h/week, irregular, 1-4 days/week with 6-20 h/week, and occasional, 1-4 day/week with $<6$ h/week); BCG: Bacillus Calmette-Guerin; QFT: QuantiFERON; TST: tuberculin skin test.

(i.e., QFT-GIT conventional, QFT-GIT borderline, TST conventional and TST borderline). Wide 95\% CIs were common and reflected sample size limitations.

\section{DISCUSSION}

Screening for LTBI using appropriate methods is important for diagnosing and prophylactically treating persons at increased risk of progression to active TB. IGRAs and TST have been proposed for serial testing in TB. To date, the main limitation for the effective investigation of contacts is the lack of a test that accurately identifies LTBI, differentiates between active and LTBI and predicts the progression to active disease. Therefore, we believe that our data could be of value for understanding the limitations of the criteria currently used to diagnose and follow-up contacts with LTBI in countries with a medium TB burden while the main issues of IGRAs to diagnose LTBI are approached.

This study is the first to report serial QFT-GIT and TST results from a cohort of close contacts of pulmo- nary TB patients in Mexico. The study primarily aimed at assessing and comparing the evolution of QFT-GIT and TST responses from household contacts after an 1824 month follow-up period, focusing specifically on the conversion and reversion rates using standard and newly proposed definitions (Ringshausen et al. 2012, Whitworth et al. 2012). Despite its limited clinical value, we elected to study the issue of TST reversion to potentially reproduce, in this Latin American population, the results that other authors have reported (Dorman et al. 2014) or commented upon (Pai \& O'Brien 2007, Trajman et al. 2013); in particular, we wanted to reproduce the observation that TST testing is prone to reversions. A secondary objective was to explore the epidemiological risk factors associated with the occurrence of conversions and reversions. Although two other studies have examined QFT instability using serial testing from household contacts (Pai et al. 2009, Shah et al. 2011), this study is the first conducted in Latin America; therefore, it adds to the body of literature on the topic. 


\section{TABLE II}

Conversion and reversion rates according to conventional and borderline zone (BZ) cut-offs assessed from two serial QuantiFERON-TB Gold In-Tube assay (QFT-GIT) and tuberculin skin test (TST) measurements among household contacts of tuberculosis (TB) patients stratified by baseline TST and QFT-GIT status, Zacatecas cohort, Mexico, 2010-2012

\begin{tabular}{|c|c|c|c|}
\hline \multirow[b]{2}{*}{ Measure/baseline status } & \multirow{2}{*}{$\begin{array}{l}\text { Change in } \\
\text { latent } \mathrm{TB} \text { status }\end{array}$} & \multicolumn{2}{|c|}{$\begin{array}{c}\text { Change from baseline to endpoint } \\
\mathrm{n}(\%)\end{array}$} \\
\hline & & Conventional $^{a}$ & $\mathrm{BZ}^{b}$ \\
\hline \multicolumn{4}{|l|}{ Change in QFT-GIT } \\
\hline \multirow{3}{*}{$\mathrm{TST} \geq 5 \mathrm{~mm}$} & Remained positive/negative & $38(76)$ & $42(84)$ \\
\hline & Conversion & $6(12)$ & $4(8)$ \\
\hline & Reversion & $6(12)$ & $4(8)$ \\
\hline \multirow[t]{3}{*}{$\mathrm{TST}<5 \mathrm{~mm}$} & Remained positive/negative & $33(91.7)$ & $33(91.7)$ \\
\hline & Conversion & $3(8.3)$ & $3(8.3)$ \\
\hline & Reversion & - & - \\
\hline \multirow[t]{3}{*}{ All contacts } & Remained positive/negative & $71(82.5)$ & $75(87.2)$ \\
\hline & Conversion & $9(10.5)$ & $7(8.1)$ \\
\hline & Reversion & $6(7)$ & $4(4.7)$ \\
\hline \multicolumn{4}{|l|}{ Change in TST } \\
\hline \multirow[t]{3}{*}{$\mathrm{QFT} \geq 0.35 \mathrm{IU} / \mathrm{mL}$} & Remained positive/negative & $29(85.3)$ & $30(88.2)$ \\
\hline & Conversion & $5(14.7)$ & $4(11.8)$ \\
\hline & Reversion & - & - \\
\hline \multirow[t]{3}{*}{$\mathrm{QFT}<0.35 \mathrm{IU} / \mathrm{mL}$} & Remained positive/negative & $29(55.8)$ & $41(78.8)$ \\
\hline & Conversion & $15(28.8)$ & $3(5.8)$ \\
\hline & Reversion & $8(15.4)$ & $8(15.4)$ \\
\hline \multirow[t]{3}{*}{ All contacts } & Remained positive/negative & $58(67.4)$ & $71(82.6)$ \\
\hline & Conversion & $20(23.3)$ & $7(8.1)$ \\
\hline & Reversion & $8(9.3)$ & $8(9.3)$ \\
\hline
\end{tabular}

$a$ : QFT-GIT conversion from $<0.35$ to $\geq 0.35$, reversion from $\geq 0.35$ to $<0.35$, TST conversion from $<5$ to $\geq 5$, reversion from $\geq 5$ to $<5$; $b$ : QFT-GIT conversion from $<0.35$ to $>0.70$, reversion from $\geq 0.35$ to $<0.20$, TST conversion from $<5$ to $>10$, reversion from $\geq 5$ to $<4$.

The main findings demonstrate relatively large conversion and reversion rates regardless of the test and definition used. As predicted, lower proportions were observed with QFT-GIT testing and when BZ definitions were used. In our opinion, the results of this work are relevant to emphasise the limited value of serial QFT measures for the follow-up of close contacts of TB patients.

Serial testing studies on household contacts are scarce (Pai et al. 2009, Shah et al. 2011); however, in accordance with our results, inexplicable conversions and the reversion rates reported are rather frequent, limiting the value of these tests to evaluate LTBI progression. We were unable to identify any factors associated with the risk of conversion or reversion in either the bivariate or the multivariate analyses. The results were often inconsistent (e.g., men had higher conversion risks according to QFT-GIT, but women had higher risks with TST when borderline cut-offs were used). Although the Indian study (Pai et al. 2009) did not explore associated factors, the study from South Africa (Shah et al. 2011) reported that older age and a baseline TST $\geq 5 \mathrm{~mm}$ were associated with QFT-GIT conversions. Additionally, female gender was related to reversions and children with baseline TST $\geq 5 \mathrm{~mm}$ were less likely to have a QFT-GIT reversion.

We conducted additional sub-analyses to explore the association between the months that had passed from baseline to follow-up testing and the probability of conversion and reversion using QFT-GIT borderline definitions, although no significant differences in the mean or median times elapsed were observed $(p>0.40)$. Even less significant differences were observed when conventional definitions were used for either the TST or QFTGIT tests $(p>0.50)$. Household clustering of conversion and/or reversion rates for the QFT-GIT were also conducted; however, the results confirm the apparent unpredictability of the results.

The boosting effect of TSTs over QFT results has been suggested as a reason that affects IGRA variability. However, the conversion rates that we observed are unlikely to be explained by this phenomenon because the boosting effect wanes within six months (Leyten et al. 2007, Baker et al. 2009, Ritz et al. 2011, Sauzullo et al. 


\section{TABLE III}

Differences in the proportions of conversion and reversion based on QuantiFERON-TB Gold In-Tube assay (QFT-GIT) and tuberculin skin test (TST) measurements using borderline zone cut-offs ${ }^{a}$ according to selected characteristics of the household contacts of tuberculosis patients, Zacatecas cohort, Mexico, 2010-2012

\begin{tabular}{|c|c|c|c|c|}
\hline \multirow[b]{3}{*}{ Selected characteristics } & \multicolumn{4}{|c|}{$\begin{array}{l}\text { Change from baseline to endpoint } \\
\mathrm{n}(\%)\end{array}$} \\
\hline & \multicolumn{2}{|c|}{ QFT-GIT } & \multicolumn{2}{|c|}{ TST } \\
\hline & Conversion & Reversion & Conversion & Reversion \\
\hline \multicolumn{5}{|l|}{ Sex } \\
\hline Male & $5(71.4)$ & $2(50)$ & $2(28.6)$ & - \\
\hline Female & $2(28.6)$ & $2(50)$ & $5(71.4)$ & $8(100)$ \\
\hline \multicolumn{5}{|l|}{ Age group (years) } \\
\hline$<30$ & $3(42.9)$ & $2(50)$ & $2(28.6)$ & $1(12.5)$ \\
\hline $30-39$ & $3(42.9)$ & $1(25)$ & $3(42.9)$ & $1(12.5)$ \\
\hline$\geq 40$ & $1(14.3)$ & $1(25)$ & $2(28.6)$ & $6(62.5)$ \\
\hline \multicolumn{5}{|l|}{$\operatorname{BMI}\left(\mathrm{kg} / \mathrm{m}^{2}\right)$} \\
\hline Overweight $(>25)$ & $4(57.1)$ & $1(25)$ & $2(28.6)$ & $5(62.5)$ \\
\hline Normal $(\leq 25)$ & $3(42.9)$ & $3(75)$ & $5(71.4)$ & $3(37.5)$ \\
\hline \multicolumn{5}{|c|}{ Exposure reported by index case ${ }^{b}$} \\
\hline $1-500 \mathrm{~h}$ in 3 months & $3(42.9)$ & - & $4(57.1)$ & $3(37.5)$ \\
\hline$>500 \mathrm{~h}$ in 3 months & $1(14.3)$ & $1(25)$ & $1(14.3)$ & $3(37.5)$ \\
\hline Undetermined & $3(42.9)$ & $3(75)$ & $2(28.6)$ & $2(25)$ \\
\hline \multicolumn{5}{|c|}{ Exposure reported by contacts ${ }^{c}$} \\
\hline Close or very close & $6(85.7)$ & $3(75)$ & $4(57.1)$ & $8(100)$ \\
\hline Irregular or occasional & $1(14.3)$ & $1(25)$ & $3(42.9)$ & - \\
\hline \multicolumn{5}{|l|}{ BCG scar } \\
\hline Yes & $5(71.4)$ & $3(75)$ & $7(100)$ & $6(75)$ \\
\hline No & $2(28.6)$ & $1(25)$ & - & $2(25)$ \\
\hline
\end{tabular}

$a$ : QFT-GIT (IU/mL) conversion from $<0.35$ to $>0.70$, reversion from $\geq 0.35$ to $<0.20$, TST $(\mathrm{mm})$ induration conversion from $<$ 5 to $>10$, reversion from $\geq 5$ to $<4 ; b$ : computed from a questionnaire applied to the index case when symptomatic and untreated (contagious period); $c$ : calculated from a questionnaire applied to the contacts after the index case was treated (non-contagious period: very close, 5-7 days/week with $\geq 30 \mathrm{~h} /$ week, close, 5-7 days/week with 21-30 h/week, irregular, 1-4 days/week with 6-20 h/week, and occasional, 1-4 days/week with $<6$ h/week); BCG: Bacillus Calmette-Guerin; BMI: body mass index.

2011, Dorman et al. 2014). However, with the available data, it is difficult to ascertain whether our findings relate to delayed immunologic responses, on-going household or community TB exposure, technical/analytical errors, inherent non-specific variations of the tests or other unknown reasons.

Serial studies on QFT and TST performance on household contacts are limited; however, the largest and longest studies on serial QFT-GIT (Slater et al. 2013) or QFT-GIT vs. TST (Dorman et al. 2014) performed in health care workers reached essentially equivalent conclusions. There was no association between reported TB exposure and conversion and more than $90 \%$ of TST converters who were retested and $75 \%$ of IGRA converters were negative on testing six months later without treatment. Those studies also assessed whether any variables would facilitate differentiation between stable and transient conversions at the time of the result. Variables related to TB exposure and IGRA quantitative values were evaluated, although none were helpful at the individual patient level.

The results of the present study should be considered in context with the relatively small sample of individuals studied and the $30 \%$ of contacts lost in the follow-up, which might have led to low statistical power and selection bias, respectively. Another limitation is the timing of the second testing, as re-exposure could have occurred during this period. Although the 18 months used between the first and second testing remain within the two-year post-infection period deemed relevant for short-term reactivations, from the clinical viewpoint, earlier conversions would have been more relevant because they are a strong risk factor for active TB progression.

The rapid expansion of IGRAs in recent years has increasingly revealed limitations and doubts, especially with regards to medical interpretation. For LTBI diag- 
nosis, these features include the clinical implications of reversion results and the meaning of the magnitude on IGRA's responses (Abubakar et al. 2013). Possible reasons for the short and long-term variabilities with IGRAs include factors that relate to the subject and those that relate to the laboratory. One possible explanation for the high proportions of IGRA conversions is that the quantitatively small changes occurring around a fixed cut-off point could result in qualitative changes from negative to positive (Diel et al. 2010, Ringshausen et al. 2010, 2012, Costa et al. 2011, Rafiza \& Rampal 2012). Some authors have suggested that the addition of a "BZ" for the QFT-GIT could help prevent misclassification of these individuals (Whitworth et al. 2012). The reasons for the intra and inter-laboratory variabilities include possible differences in laboratory procedures, such as variations in processing, time to incubation and incubation time (Doberne et al. 2011, Whitworth et al. 2012).

As mentioned previously, discordance on QFT-GIT serial testing is a concern that has no consensus explanation and can be significantly higher in patients with a low mitogen response (Woo et al. 2014). Otherwise, discordance between LTBI tests might have resulted from intermittent antigen secretion from Mycobacterium tuberculosis, as suggested previously (Hill et al. 2007a, Park et al. 2012). However, we must consider that IGRAs are highly dynamic tests and $\mathrm{T}$ cell responses tend to fluctuate according to the variable status of TB infection (Pai et al. 2006). QFT-GIT reversions might reflect the clearance of TB infection or biologic variability, particularly when the results of the baseline TST are discordant. Evidence demonstrates that children with discordant test results display a mixed biomarkers profile, with activation of both pro and anti-inflammatory markers compared with children with concordant results for TST and QFT-GIT (Dhanasekaran et al. 2014); therefore, the TST and/or QFT-GIT positivity reflect different, but overlapping aspects of host immunity. Further longitudinal studies of serial $\mathrm{T}$ cell responses and active TB progression are required to clarify the variable IGRA results.

The substantial incidence of TB disease within five years after exposure and particularly within the first year, emphasises the importance of serial screening for TB in contacts that do not receive prophylactic treatment for LTBI (Fox et al. 2013). However, our data in high-risk groups of Mexicans suggest that the QFTGIT offers little advantage over the TST for assessing LTBI prevalence (Serrano-Escobedo et al. 2013) and for the follow-up of close contacts. It remains important to inform contacts about their on-going risk of developing $\mathrm{TB}$, even after the index case has been treated and to improve referral strategies if symptoms appear (Fox et al. 2013) while better testing tools are developed.

At present, the Mexican Official Norm states that TB chemoprophylaxis should be given as follows: (i) for six months to contacts $<5$ years with or without $B C G$ vaccination, (ii) for six months to those aged five-14 years with no BCG in whom TB has been ruled out, (iii) to contacts aged $\geq 15$ years who are human immunodeficiency virus-positive or have another immunosuppressive event during the initial 12 months after having ruled out pulmonary or extra-pulmonary TB and (iv) $10 \mathrm{mg} / \mathrm{kg}$ of isoniazid up to $300 \mathrm{mg} /$ day should be given orally under strict supervision. Although the results of this study have limited value in Mexico because there is no official endorsement for the provision of preventive treatment for adult healthy LTBI individuals, these results could be of relevance in other settings where prophylaxis is in use.

Despite the small sample size, which might have affected the precision of the estimates and the $30 \%$ of subjects lost to follow-up, which might have led to selection bias, we believe that the relatively large reversion rates, the poor internal logic across methods and definitions and the lack of statistically significant factors associated with the incidence of conversions and reversions make the results of serial testing difficult to interpret. To date, neither test appears to be adequate and/or accurate for diagnosing LTBI. The inconsistency and apparent randomness of the results are perplexing and add to the limitations in the effectiveness of these methods and to the definitions of follow-up individuals in close contact with TB patients.

\section{ACKNOWLEDGEMENTS}

To the medical staff and laboratory personnel Yannin de la Torre Mercado, Marco Antonio Ríos Ceballos and Yolanda García de Lira.

\section{REFERENCES}

Abubakar I, Griffiths C, Ormerod P 2012. Diagnosis of active and latent tuberculosis: summary of NICE guidance. BMJ 345: e6828.

Abubakar I, Stagg HR, Whitworth H, Lalvani A 2013. How should I interpret an interferon gamma release assay result for tuberculosis infection? Thorax 68: 298-301.

Baker CA, Thomas W, Stauffer WM, Peterson PK, Tsukayama DT 2009. Serial testing of refugees for latent tuberculosis using the QuantiFERON Gold In-Tube: effects of an antecedent tuberculin skin test. Am J Trop Med Hyg 80: 628-633.

Costa JT, Silva R, Sá R, Cardoso MJ, Nienhaus A 2011. Serial testing with the interferon-gamma release assay in Portuguese healthcare workers. Int Arch Occup Environ Health 84: 461-469.

Dhanasekaran S, Jenum S, Stavrum R, Ritz C, Kenneth J, Vaz M, Doherty TM, Grewal HM 2014. Concordant or discordant results by the tuberculin skin test and the QuantiFERON-TB test in children reflect immune biomarker profiles. Genes Immun 15: 265-274.

Diel R, Loddenkemper R, Nienhaus A 2010. Evidence-based comparison of commercial interferon-gamma release assays for detecting active TB: a meta-analysis. Chest 137: 952-968.

Doberne D, Gaur RL, Banaei N 2011. Pre-analytical delay reduces sensitivity of QuantiFERON-TB Gold In-Tube assay for detection of latent tuberculosis infection. J Clin Microbiol 49: 3061-3064.

Dorman SE, Belknap R, Graviss EA, Reves R, Schluger N, Weinfurter P, Wang Y, Cronin W, Hirsch-Moverman Y, Teeter LD, Parker M, Garrett DO, Daley CL 2014. Interferon-gamma release assays and tuberculin skin testing for diagnosis of latent tuberculosis infection in healthcare workers in the United States. Am J Respir Crit Care Med 189: 77-87.

Ewer K, Millington KA, Deeks JJ, Alvarez L, Bryant G, Lalvani A 2006. Dynamic antigen-specific T-cell responses after pointsource exposure to Mycobacterium tuberculosis. Am J Respir Crit Care Med 174: 831-839. 
Fox GJ, Barry SE, Britton WJ, Marks GB 2013. Contact investigation for tuberculosis: a systematic review and meta-analysis. Eur Respir J 41: 140-156.

Franken WP, Koster BF, Bossink AW, Thijsen SF, Bouwman JJ, van Dissel JT, Arend SM 2007. Follow-up study of tuberculosis-exposed supermarket customers with negative tuberculin skin test results in association with positive gamma interferon release assay results. Clin Vaccine Immunol 14: 1239-1241.

Friedman LN, Nash ER, Bryant J, Henry S, Shi J, D’Amato J, Khaled GH, Russi MB, O’Connor PG, Edberg SC, Pisani MA, Cain HC, Tanoue L, Weissman DN 2006. High rate of negative results of tuberculin and QuantiFERON tests among individuals with a history of positive skin test results. Infect Control Hosp Epidemiol 27: 436-441.

Hill PC, Brookes RH, Fox A, Jackson-Sillah D, Jeffries DJ, Lugos MD, Donkor SA, Adetifa IM, de Jong BC, Aiken AM, Adegbola RA, McAdam KP 2007a. Longitudinal assessment of an ELISPOT test for Mycobacterium tuberculosis infection. PLoS Med 4: e192.

Hill PC, Jeffries DJ, Brookes RH, Fox A, Jackson-Sillah D, Lugos MD, Donkor SA, de Jong BC, Corrah T, Adegbola RA, McAdam KP 2007b. Using ELISPOT to expose false positive skin test conversion in tuberculosis contacts. PLOS ONE 2: e183.

Lee SS, Liu YC, Huang TS, Chen YS, Tsai HC, Wann SR, Lin HH 2008. Comparison of the interferon-gamma release assay and the tuberculin skin test for contact investigation of tuberculosis in BCGvaccinated health care workers. Scand J Infect Dis 40: 373-380.

Leyten EM, Prins C, Bossink AW, Thijsen S, Ottenhoff TH, van Dissel JT, Arend SM 2007. Effect of tuberculin skin testing on a $M y$ cobacterium tuberculosis-specific interferon-gamma assay. Eur Respir J 29: 1212-1216.

Mazurek GH, Jereb J, Lobue P, Iademarco MF, Metchock B, Vernon A 2005. Guidelines for using the QuantiFERON-TB Gold test for detecting Mycobacterium tuberculosis infection, United States. MMWR Recomm Rep 54: 49-55.

Mazurek GH, Jereb J, Vernon A, LoBue P, Goldberg S, Castro K 2010. Updated guidelines for using interferon gamma release assays to detect Mycobacterium tuberculosis infection - United States, 2010. MMWR Recomm Rep 59: 1-25.

Menzies D 1999. Interpretation of repeated tuberculin tests. Boosting, conversion and reversion. Am J Respir Crit Care Med 159: 15-21.

Moon HW, Hur M 2013. Interferon-gamma release assays for the diagnosis of latent tuberculosis infection: an updated review. Ann Clin Lab Sci 43: 221-229.

Pai M, Dheda K, Cunningham J, Scano F, O’Brien R 2007. T-cell assays for the diagnosis of latent tuberculosis infection: moving the research agenda forward. Lancet Infect Dis 7: 428-438.

Pai M, Joshi R, Dogra S, Mendiratta DK, Narang P, Kalantri S, Reingold AL, Colford Jr JM, Riley LW, Menzies D 2006. Serial testing of health care workers for tuberculosis using interferongamma assay. Am J Respir Crit Care Med 174: 349-355.

Pai M, Joshi R, Dogra S, Zwerling AA, Gajalakshmi D, Goswami K, Reddy MV, Kalantri A, Hill PC, Menzies D, Hopewell PC
2009. T-cell assay conversions and reversions among household contacts of tuberculosis patients in rural India. Int J Tuberc Lung Dis 13: 84-92.

Pai M, O'Brien R 2007. Serial testing for tuberculosis: can we make sense of T cell assay conversions and reversions? PLoS Med 4: e208.

Park JS, Lee JS, Kim MY, Lee CH, Yoon HI, Lee SM, Yoo CG, Kim YW, Han SK, Yim JJ 2012. Monthly follow-ups of interferongamma release assays among health-care workers in contact with patients with TB. Chest 142: 1461-1468.

Rafiza S, Rampal KG 2012. Serial testing of Malaysian health care workers with QuantiFERON(R)-TB Gold In-Tube. Int $J$ Tuberc Lung Dis 16: 163-168.

Ringshausen FC, Nienhaus A, Schablon A, Schlosser S, SchultzeWerninghaus G, Rohde G 2010. Predictors of persistently positive Mycobacterium-tuberculosis-specific interferon-gamma responses in the serial testing of health care workers. BMC Infect Dis 10: 220.

Ringshausen FC, Schablon A, Nienhaus A 2012. Interferon-gamma release assays for the tuberculosis serial testing of health care workers: a systematic review. J Occup Med Toxicol 7: 6.

Ritz N, Yau C, Connell TG, Tebruegge M, Leslie D, Curtis N 2011. Absence of interferon-gamma release assay conversion following tuberculin skin testing. Int J Tuberc Lung Dis 15: 767-769.

Sauzullo I, Massetti AP, Mengoni F, Rossi R, Lichtner M, Ajassa C, Vullo V, Mastroianni CM 2011. Influence of previous tuberculin skin test on serial IFN-gamma release assays. Tuberculosis (Edinb) 91: 322-326.

Serrano-Escobedo CJ, Enciso-Moreno JA, Monarrez-Espino J 2013. Performance of tuberculin skin test compared to QFT-IT to detect latent TB among high-risk contacts in Mexico. Arch Med Res 44: $242-248$.

Shah M, Kasambira TS, Adrian PV, Madhi SA, Martinson NA, Dorman SE 2011. Longitudinal analysis of QuantiFERON-TB Gold In-Tube in children with adult household tuberculosis contact in South Africa: a prospective cohort study. PLOS ONE 6: e26787.

Slater ML, Welland G, Pai M, Parsonnet J, Banaei N 2013. Challenges with QuantiFERON-TB Gold assay for large-scale, routine screening of US healthcare workers. Am J Respir Crit Care Med 188: $1005-1010$.

Trajman A, Steffen RE, Menzies D 2013. Interferon-gamma release assays versus tuberculin skin testing for the diagnosis of latent tuberculosis infection: an overview of the evidence. Pulm Med 2013: 601737 .

Whitworth WC, Hamilton LR, Goodwin DJ, Barrera C, West KB, Racster L, Daniels LJ, Chuke SO, Campbell BH, Bohanon J, Jaffar AT, Drane W, Maserang D, Mazurek GH 2012. Withinsubject interlaboratory variability of QuantiFERON-TB Gold InTube tests. PLoS ONE 7: e43790.

Woo KS, Choi JL, Kim BR, Kim JE, Kim BG, Lee H, Kim KH 2014. Significance of interferon-gamma response to mitogen in serial QuantiFERON-TB Gold In-Tube assay of routine laboratory practice. Clin Chim Acta 430: 79-83. 